\title{
Solvent effects on the properties of
}

\section{hyperbranched polythiophenes}

Juan Torras, ${ }^{1,}$ David Zanuy, ${ }^{2}$ David Aradilla, ${ }^{2,3, \diamond}$ and Carlos

\begin{abstract}
Alemán ${ }^{2,3, *}$
${ }^{1}$ Departament d'Enginyeria Química, EEI, Universitat Politècnica de Catalunya, Av. Pla de la Massa, 8, 08700 Igualada, Spain.

${ }^{2}$ Departament d'Enginyeria Quimica, E.T.S. d'Enginyeria Industrial de Barcelona, Universitat Politècnica de Catalunya, Diagonal 647, 08028 Barcelona, Spain.

${ }^{3}$ Center for Research in Nano-Engineering, Universitat Politècnica de Catalunya, Campus Sud, Edifici C', C/Pasqual i Vila s/n, 08028 Barcelona, Spain.
\end{abstract}

*Corresponding authors: joan.torras@upc.edu and carlos.aleman@upc.edu

${ }^{\diamond}$ Current address: CEA, INAC-SPRAM, 17 rue des Martyrs F-38000 Grenoble, France ce 


\begin{abstract}
The structural and electronic properties in solution of all-thiophene dendrimers and dendrons have been evaluated using very different theoretical approaches based on quantum mechanical (QM) and hybrid $\mathrm{QM} /$ molecular mechanics (MM) methodologies: i) calculations on minimum energy conformations using an implicit solvation model combined with density functional theory (DFT) or time-dependent DFT methods (TDDFT); ii) hybrid QM/MM calculations, in which the solute and solvent molecules are represented at the DFT level using as point charges, respectively, on snapshots extracted from classical molecular dynamics (MD) simulations with explicit solvent molecules; and iii) QM/MM-MD trajectories in which the solute is described at the DFT or TDDFT level and the explicit solvent molecules are represented using classical force-fields. Calculations have been performed on dichloromethane, tetrahydrofurane and dimethylformamide. Comparison of the results obtained using the different approaches with available experimental data indicates that, the incorporation of effects associated to both the conformational dynamics of the dendrimer and the explicit solvent molecules is strictly necessary to satisfactorily reproduce the properties of the investigated systems. Accordingly, QM/MM-MD simulations are able to capture such effects providing a reliable description of electronic properties-conformational flexibility relationships in all-Th dendrimers.
\end{abstract}




\section{INTRODUCTION}

Since their discovery in the late $70{ }^{\prime} \mathrm{s}^{1-3}$ the research interest for dendrimers, which are virtually monodisperse hyperbranched macromolecules, has gained an increasing interest because of their broad range of applications in, for example, supramolecular chemistry, ${ }^{4-6}$ self-assembly processes, ${ }^{7-9}$ anticancer therapies ${ }^{10-12}$ and diagnostic imaging. ${ }^{13-15}$ The structure of dendrimers is divided into three distinct regions: the core, branched repeat units emanating from this core, and end-groups of the outer layer of repeat units (Figure 1a). ${ }^{16}$ The number of concentric layers of branching points is known as the generation of the dendrimer. It is worth noting that the amount of end groups in dendrimers grows exponentially with the generation. Dendrimers undergo changes in size, shape and flexibility as a function of increasing generation. ${ }^{16-19}$

On the other hand, dendrons are monodisperse dendritic wedge-shaped structures that comprise a single function at the core and multifunctionality at the outer layer (periphery). Figure 1a illustrates the difference between dendrimers and dendrons structures. As it can be seen, the former dendritic structures usually contain two or more dendrons coupled together to a core moiety.

All-thiophene (Th) based dendrimers and dendrons are considered as very promising kinds of conducting materials with different energy-related applications. ${ }^{20}$ Since Advincula and co-workers reported on the first Th dendrimer synthesis (up to $30 \mathrm{Th}$ rings), ${ }^{21,22}$ advances in synthetic approaches enabled the preparation of increasingly larger. Thus, Bäuerle and co-workers ${ }^{23}$ synthesized different all-Th dendrimers containing up to $90 \mathrm{Th}$ rings with a divergent/convergent approach to facilitate the inclusion of functionalities in the external surface of the conducting dendrimer. More recently, the same group reported a synthetic approach to a series of generational 2,2':3',2''-terthiophene-based Th dendrons and dendrimers. ${ }^{24}$ 
$\pi$-Conjugated dendrimers are considered attractive candidates for organic-electronic applications due to their solution processability, redox properties, band gap $\left(\varepsilon_{\mathrm{g}}\right)$ and isotropic charge-transport ability. ${ }^{25}$ In the particular, all-Th dendrons and dendrimers, hereafter denoted $n \mathrm{~T}$ (where $n$ is the number of Th rings), behave as p-type semiconductors, being proposed as components of photovoltaic devices and organic solar cells. ${ }^{24,26,27}$ Therefore, understanding of the electronic properties of $n \mathrm{~T}$ is a key stage not only in the ongoing evolution of these systems for their promising applications but also in the development of new alternatives to conventional oligothiophenes and polythiophenes in the organic semiconductor field. The ionization potential (IP) and $\varepsilon_{\mathrm{g}}$ of $n \mathrm{~T}$ (with $n=3,9,21$ and 45 for dendrons and $n=6,18,42,90$ for dendrimers) were recently investigated in solution using cyclic voltammetry (CV) and UV-vis spectroscopy. ${ }^{24}$

Given the importance of the structural and electronic properties in the application of all-Th dendrimers, theoretical studies based on quantum mechanics are necessary not only to provide comprehensive understanding at the microscopic level but also to gain deeper insight on the intrinsic characteristics of these materials. Within this context, we used quantum mechanical (QM) calculations to study the structural and electronic properties of $n \mathrm{~T}$ dendrimers and dendrons with $n=3,6,7,9,14,15,18,21,30,42$ and 45 in $_{\text {vacuum. }}{ }^{28,29}$ More recently, we also examined the structural and electronic properties of $3 \mathrm{~T}$ and $7 \mathrm{~T}$ dendrons attached to a phenyl core, ${ }^{30}$ which had been used in a pioneering work by Schlüter and co-workers ${ }^{31}$ to synthesize Th-containing second and third generation dendronized macromonomers with methacrylate polymerizable units as well as their corresponding dendronized polymers. Although QM calculations provided important qualitative information, in all cases electronic properties predicted in vacuum exhibit deviations with respect to experimental estimations obtained in solution. ${ }^{24,31}$ 
Although the latter may be attributed to the influence of the environment in the IP and $\varepsilon_{\mathrm{g}}$, other important factors, as the conformational flexibility and dynamics may be also responsible of such deviations. Thus, QM predictions were performed considering the energy minimized conformations of the dendrons and dendrimers, the effects associated to the conformational variability being neglected from our theoretical predictions. ${ }^{28-30}$

In this work we re-visit the structural and electronic properties of all-Th dendrimers and dendrons but considering the influence of the both the solvent and the conformational variability. More specifically, we have examined the influence of the medium on the IP, $\varepsilon_{\mathrm{g}}$, structural dynamics and conformational flexibility by considering dichloromethane (DCM), tetrahydrofurane (THF) and dimethylformamide (DMF) as solvents. In addition, each of such factors have been analysed considering different theoretical approaches, allowing us to properly identify the physical features that affect the properties of such unique systems. More specifically, theoretical predictions have been carried out using: a) QM calculations using an implicit solvent model combined with density functional theory (DFT) and time dependent density functional theory (TDDFT) methodologies; b) single point quantum mechanics/molecular mechanics (QM/MM) calculations using snapshots extracted from classical molecular dynamics (MD) simulations using explicit solvent molecules; and c) QM/MM-MD simulations with explicit solvent molecules. Amazingly, despite the huge amount of theoretical investigations on dendrimers, the number of QM/MM and/or QM/MM-MD studies is very scarce and, in addition, the role of the solvent was neglected in all them. More specifically, Rodríguez-Ropero et al. ${ }^{32}$ studied the assembly of up to five 30 T molecules using static QM/MM calculations, while Roitberg and co-workers ${ }^{33}$ used QM/MM-MD simulations to predict the electronic spectra at different temperatures of a nanostar dendrimer made of phenylene-ethylene dendrimer attached to a ethynylperylene 
chromophore. Results obtained in this work prove that the microfluctuations induced by different types of solvents as well as their effects on the evolution of the electronic properties are satisfactorily captured by QM/MM-MD trajectories.

\section{METHODS}

All QM and QM/MM calculations were carried out using the Gaussian 09 computer package. ${ }^{34}$ The parameters used for the structural analysis are defined in Figure $1 \mathrm{~b}$ for 6T. These consists in bond distances $\left(R_{D-D}, R_{\alpha-\alpha}\right.$ and $\left.R_{\alpha-\beta}\right)$ and dihedral angles $\left(\theta_{D-D}, \theta_{\alpha-}\right.$ ${ }_{\alpha}$ and $\left.\theta_{\alpha-\beta}\right)$ associated to the rotation of such bonds.

QM calculations. The effect of the implicit solvent in the electronic properties of $n \mathrm{~T}$ dendrons $(n=3,9,21$ and 45) and dendrimers $(n=6,18,42$ and 90), which are depicted in Figure S1, was estimated using the Polarizable Continuum Model (PCM) developed by Tomasi and co-workers. ${ }^{35-37}$ This model represents the polarization of the liquid by a charge density appearing on the surface of the molecular-shaped cavity created in the solvent. The magnitude of these charges is proportional to the derivative of the solute electrostatic potential at each point calculated from the molecular wavefunction. Point charges are included in the one-electron Hamiltonian inducing polarization of the solute, an iterative calculation being applied until the wavefunction and the surface charges are self-consistent. PCM calculations were performed at the B3LYP/6-31G(d) level using the standard protocol. The influence of the bulk solvent properties have been examined considering DCM $(\varepsilon=8.93)$, THF $(\varepsilon=7.4257)$ and DMF $(\varepsilon=37.219)$ in the PCM calculations. As the DMF solvent is not defined in the standard version of Gaussian 09, the parameterization of the PCM for predicting the properties of the solute in this solvent were taken from Böes et al. ${ }^{38}$ Starting geometries were taken from our previous work, ${ }^{29}$ in which complete geometry optimizations of $n \mathrm{~T}$ were carried in vacuum using 
the B3LYP functional ${ }^{39,40}$ combined with the $6-31 \mathrm{G}(\mathrm{d})^{41}$ and $6-311++\mathrm{G}(\mathrm{d}, \mathrm{p})^{42,43}$ basis sets (i.e. B3LYP/6-31G(d) and B3LYP/6-311++G(d,p) level, respectively). Thus, it has been largely proved that solvent-induced changes in bond lengths and angles have a very small influence on the free energy of solvation, $\Delta \mathrm{G}_{\mathrm{sol}}$ (i.e. solute geometry relaxations in solution and single point calculations on the gas-phase optimized geometries provide almost identical values of $\left.\Delta \mathrm{G}_{\mathrm{sol}}\right) .{ }^{44-46}$

The IPs were determined using the Koopmans' theorem, according to which the IP is taken as the negative of the highest occupied molecular orbital (HOMO) energy (i.e. $\left.\mathrm{IP}^{\mathrm{KT}}=-\varepsilon_{\mathrm{HOMO}}\right)$. Although Koopman's theorem does not apply to DFT and the energies of Kohn-Sham orbitals do not involve any physical meaning, Janak's theorem ${ }^{47}$ was used by Perdew ${ }^{48}$ to show the connection between the IP and $\varepsilon_{\text {HOMO. The }} \varepsilon_{\mathrm{g}}$ was estimated using two different strategies. In the first one, $\varepsilon_{\mathrm{g}}$ was approximated as the difference between the energies of the frontier orbitals: $\varepsilon_{\mathrm{g}}=\varepsilon_{\mathrm{LUMO}}-\varepsilon_{\mathrm{HOMO}}$. In an early work, Levy and Nagy showed that in DFT calculations $\varepsilon_{\mathrm{g}}$ can be correctly estimated using this procedure. ${ }^{49}$ The second estimation of $\varepsilon_{\mathrm{g}}$ was derived from the excitation energies calculated with TD-DFT. This method, which is widely applied to study the UV-vis spectra of conjugated organic compounds, provides a robust and efficient description of the low-lying molecular states. ${ }^{50-52}$ Electronic excitations were evaluated combining the PCM framework with the B3P86/6-31G(d) ${ }^{53,54}$ level. In all cases the $\varepsilon_{\mathrm{g}}$ was extracted from the first low-lying transition with a large oscillator strength.

MD simulations. A series of MD simulations were performed to study the solvation of $6 \mathrm{~T}$ and $18 \mathrm{~T}$ dendrimers in DCM, THF and DMF. All MD trajectories were generated using the scalable computer package NAMD ${ }^{55}$ and the AMBER force-field. ${ }^{56}$ Stretching and bending equilibrium parameters for $6 \mathrm{~T}$ and $18 \mathrm{~T}$ dendrimers were derived from quantum mechanical calculations at the B3LYP/6-31G(d) level. The force-field 
parameters for the DCM, THF and DMF solvents were taken from Blas et al., ${ }^{57}$ Rodríguez-Ropero et al. ${ }^{58}$ and Shih et al., ${ }^{59}$ respectively, while the rest of force-field parameters, with the obvious exception of the electrostatic charges, were adapted from the $\mathrm{AMBER}^{56}$ and Generalized AMBER force field ${ }^{60}$ (GAFF) libraries. Atomic charges were obtained using the Restrained ElectroStatic Potential (RESP) strategy $^{61}$ at the HF/6-31G(d) level. The electrostatic parameters derived using this procedure are fully compatible with the current AMBER and GAFF force-fields. The number of explicit DCM, THF and DMF solvent molecules in 6T / 18T simulations was 2113 / 2083, 2101 / 2070 and 2265 / 2237, respectively.

All MD simulations were performed using the NVT ensemble in a cubic simulation box. Periodic boundary conditions were applied using the nearest image convention. The box size was adjusted to fit the complex size, the initial box dimensions being $\left(66 \times 66 \times 66 \AA^{3}\right)$ to ensure infinite dilution. Before each simulation was run, the potential energy of each system was minimized using 5000 conjugate gradient steps. To ensure a uniform distribution of the dendrimer in solution, the system was heated to $500 \mathrm{~K}$ and after a cooling was carried out to $298 \mathrm{~K}$ employing NPT conditions. A total time of 100 ns was employed for each system.

Single point QM/MM calculations. Single point $\mathrm{QM} / \mathrm{MM}$ calculations performed for $6 \mathrm{~T}$ and $18 \mathrm{~T}$ dendrimers in DCM, THF and DMF solutions using selected snapshots extracted from classical MD simulations. While the dendrimer was described at the QM B3LYP/6-31G(d) level, the solvent molecules were represented as point charges. The IP and the $\varepsilon_{\mathrm{g}}$ were evaluated using the procedure described previously for PCM-DFT calculations. For each dendrimer and solvent, calculations were performed over 10 snapshots that were regularly extracted from the corresponding MD trajectory (i.e. every $10 \mathrm{~ns})$. 
QM/MM-MD calculations. Hybrid QM/MM-MD calculations were carried out for 6T and 18T with explicit DCM, THF or DMF solvent molecules to examine the influence of dynamical effects in the electronic structure of solvated dendrimers. For this purpose, dendrimers were treated at the QM level while the solvent molecules were considered classically. For each system, the last snapshot of the corresponding classical MD simulation was used as starting geometry of the hybrid QM/MM-MD trajectory.

All QM/MM-MD simulations were performed using the Amber-PUPIL-Gaussian interface. $^{62}$ The AMBER program ${ }^{63}$ was performing the task of classical engine connected through the PUPIL interface with the Gaussian 09 program, ${ }^{34}$ which took the role of quantum engine within the QM/MM-MD approach. The quantum zone (QZ) includes only the dendrimer molecule, and two different levels of calculation were considered to obtain final QM/MM-MD trajectories: Initially, a trajectory of 20 ps was obtained by considering the QZ at B3LYP/3-21G(d) level. Finally, last 5 ps of the previous trajectory were recalculated to obtain the electronic properties using singlepoint TD-DFT calculations at the B3P86/6-31G(d) level.

Periodic boundary conditions were applied using the nearest image convention. Before each production simulation, a classical equilibration protocol, similar to that described above for classical MD simulations, was applied. Once equilibrated, an initial heating was carried out at $298 \mathrm{~K}$ employing NPT conditions during 2 ps with a time step of 0.5 fs. Finally, 20 ps of NPT production trajectory were performed at $298 \mathrm{~K}$ using a time step of 0.5 fs. Data were stored each 2 fs for subsequent structural statistics. The last 5 ps of each trajectory were post processed using Amber-PUPIL-Gaussian interface to obtain accurate electronic properties using the TD-DFT methodology.

\section{RESULTS AND DISCUSSION}


Figures $2 \mathrm{a}$ and $2 \mathrm{~b}$ represent the variation of the IP and $\varepsilon_{\mathrm{g}}$, respectively, calculated in DCM for $n \mathrm{~T}$ dendrons and dendrimers at the PCM-B3LYP/631G(d) level (hereafter denoted PCM-DFT) against $1 / n$. Although the IP decreases with $n$ for both architectures, this variation does not follow the typical linear behaviour usually observed for linear oligothiophenes (especially for those with $n \leq 12$ ),${ }^{64}$ evidencing the influence of the molecular architecture (i.e. the ratio between $\alpha-\alpha$ and $\alpha-\beta$ linkages). Comparison with the IPs determined by $\mathrm{CV}^{24}$ shows that theoretical values are underestimated by $0.4-0.7 \mathrm{eV}(\sim 10 \%)$, which should be partially attributed to the electronic and geometric relaxations of the oxidized species neglected by the Koopman theorem. In contrast, PCM-DFT $\varepsilon_{\mathrm{g}}$ values are overestimated by $\sim 10 \%(0.3-0.5 \mathrm{eV})$ with respect to the experimental measures, even though both sets of values follow the same behaviour (i.e. $\varepsilon_{\mathrm{g}}$ rapidly decreases with $n$ ). This deviation decreases to $\sim 5 \%$ for PCMTD-DFT-B3P86/6-31G(d) (hereafter PCM-TDDFT) values, indicating that the TD-DFT method systematically corrects the overestimation produced by the DFT method (Figure $2 b)$.

Comparison between the IP and $\varepsilon_{\mathrm{g}}$ values calculated in dichloromethane with those estimated in the gas-phase, ${ }^{28,29}$ which were obtained at a very similar level of theory (i.e. B3LYP/6-31G(d) for both DFT and TD-DFT), is displayed in Figure S2. The average deviation between the obtained in solution and in the gas-phase is of only 0.07 $\mathrm{eV}$ for the IP and of $0.05 \mathrm{eV}$ for $\varepsilon_{\mathrm{g}}$ (both DFT and TD-DFT). These results suggest that the influence of the DCM polarization effects in such electronic properties is very small. Moreover, PCM-DFT and PCM-TD-DFT calculations considering THF and DMF solvents led to IP and $\varepsilon_{\mathrm{g}}$ values practically identical to those obtained in DCM (i.e. deviations were lower than $0.1 \mathrm{eV}$ in all cases) corroborating the small influence of the solvent polarization effects. This feature is supported by the large resemblance among 
the frontier orbitals calculated in the gas-phase and in DCM, THF and DMF solutions, as is illustrated in Figures 3 and S3 for 6T and 18T, respectively. As it can be seen, the HOMO / LUMO of these dendrimers is mainly /exclusively delocalized along the segment involving Th rings connected by $\alpha-\alpha$ linkages, independently of the environment.

In order to analyse the structure and organization of solvent molecules around $n \mathrm{~T}$ dendrimers, classical MD trajectories of representative dendrimers were carried out using explicit solvent molecules. Thus, $100 \mathrm{~ns}$ production trajectories were performed for $6 \mathrm{~T}$ and $18 \mathrm{~T}$ considering DCM, THF and DMF solvents (i.e. $100 \mathrm{~ns} \times 2$ dendrimers $\times$ 3 solvents $=600 \mathrm{~ns}$ ). For each trajectory, the radial probability distribution functions of each solvent molecule as a function of the geometric centre of each Th ring, $g(r)$, were calculated considering different references: (i) the centre of masses of the DCM, THF or DMF solvent molecule; and (ii) characteristic atoms of the solvent molecule (i.e. chlorine and carbon for DCM, oxygen and carbon for THF, and nitrogen and oxygen for DMF). Figures 4 and S4 depicts the $g(r)$ calculated for $6 \mathrm{~T}$ and $18 \mathrm{~T}$, respectively. Interestingly, linear-like profiles were obtained in all cases indicating that, independently of both the solvent and the reference used to evaluate the $g(r), n \mathrm{~T}$ dendrimers do not exhibit any preferred organization of the solvent molecules located at the first and second solvation shells. Thus, no peak is observed at low distances for both $6 \mathrm{~T}$ and $18 \mathrm{~T}$.

The efficacy of the conformational sampling was proved by examining the variability of a very simple electronic property, the charge on every Th ring, along the $100 \mathrm{~ns}$ MD trajectory. The charge of the Th ring was expressed as the sum of the molecular electrostatic potential (MEP) derived atomic charges for all atoms of a given ring in a given snapshot. For this purpose, MEP derived atomic charges were calculated for $6 \mathrm{~T}$ 
and 18T geometries extracted from snapshots taken from their corresponding simulations in DCM at $0.4 \mathrm{~ns}$ intervals (i.e. 250 snapshot were considered for each dendrimer). Interestingly, we observed that the charge of the Th ring fluctuates between approximately -0.15 and 0.20 e.u. Moreover, these limit values, which were similar for both $6 \mathrm{~T}$ and $18 \mathrm{~T}$, were reached multiple times during the MD simulation corroborating that the conformational flexibility of the dendrimers was satisfactorily described during the $100 \mathrm{~ns}$ MD simulations.

Of course, there are some electronic properties that change with the conformation. The simplest one, which has been used to follow the conformational changes during the MD simulations, corresponds to the charge on each thiophene ring. This was evaluated by summing the molecular electrostatic potential derived atomic charges for all atoms of a given ring in a given snapshot. Our results indicate that for both 6T and 18 the charge of each thiophene ring varies between approximately -0.15 and 0.20 e.u. during the MD simulation evidencing not only the impact of the conformational but also the reliability of the sampling since such two extreme values were reached multiple times during the $100 \mathrm{~ns}$ trajectories.

On the other hand, apparently the influence of the solvent in the molecular conformation of $6 \mathrm{~T}$ and $18 \mathrm{~T}$ is not negligible. This is proved in Figure 5, which displays superimposed structures of $18 \mathrm{~T}$ taken from MD trajectories in DCM, THF and DMF at different time intervals (i.e. 5, 50 and $100 \mathrm{~ns}$ ). As it can be seen, the solvent causes structural fluctuations at the end of the branches. These fluctuations have been attributed to the breathing motions induced by thermal effects on both the solute and the solvent molecules. Moreover, the fluctuations of the branches in the dendrimers are observed for all solvents, independently of their polarity (Figure S5). Obviously, breathing motions are less pronounced in 6T since the former dendrimer results from 
the combination of only two dendrons. Accordingly, the extension of the fluctuations in terms of length-scale is significantly smaller for 6T than for 18T (Figure S6).

Single point QM/MM calculations, in which atoms belonging to solvent molecules were represented as electrostatic charges, were performed over 10 snapshots that were extracted every $10 \mathrm{~ns}$ from the classical MD trajectories. These calculations allowed us to include explicitly the electrostatic effects caused by the different solvation shells in the evaluation of the electronic properties of $6 \mathrm{~T}$ and 18T. Results obtained in DCM solution are compared in Table 1 with those derived from QM calculations. As it can be seen, the $\varepsilon_{\mathrm{g}}$ and IP derived from $\mathrm{QM} / \mathrm{MM}$ are closer to the experimental values than PCM-DFT estimations, even though both PCM-DFT and QM/MM calculations were performed at the B3LYP/6-31G(d) level. Indeed, QM/MM results are pretty similar to those achieved using the PCM-TD-DFT calculations, suggesting that electrostatic effects associated to explicit solvent molecules artificially corrects the omission of excited states in DFT. As occurred for QM calculations, the electronic properties obtained in THF and DMF solutions were very similar to those reached in DCM.

Hybrid QM/MM-MD simulations on $6 \mathrm{~T}$ and $18 \mathrm{~T}$ were performed to simultaneously consider all effects that may affect the structural and electronic properties of such dendrimers. More specifically, QM/MM-MD calculations allowed us to include the influence of: (i) bulk solvent effects since all solvent molecules are explicitly represented; (ii) specific and non-specific dendrimer-solvent interactions at the first solvation shell, both electrostatic and van der Waals intermolecular interactions being explicitly considered; (iii) the dynamics of the solvent and the dendrimer at room temperature through the MD; and (iv) the excited states since the TD-DFT methodology was used to describe the dendrimer at the QM level. 
Structural parameters derived from QM/MM-MD simulations in solution and from QM calculations in both the gas-phase and DCM for the two dendrimers are compared in Table 2. Although QM geometry optimizations predict very similar bond lengths for $6 \mathrm{~T}$ and $18 \mathrm{~T}$, independently of the environment, small but significant differences are detected upon the incorporation of dynamical effects through hybrid QM/MM-MD simulations. Thus, in general $R_{\alpha-\alpha}, R_{\alpha-\beta}$ and $R_{D-D}$ (Figure $1 b$ ) are slightly higher for $6 \mathrm{~T}$ than $18 \mathrm{~T}$, independently of the solvent polarity. This feature, which is not captured in optimized geometries, should be attributed to the fact that the rigidity of dendronized structures increases with the generation number. ${ }^{64-67}$ Accordingly, the small elongations at bond lengths caused by the dynamic and thermal effects become less appreciable in $18 \mathrm{~T}$ than in $6 \mathrm{~T}$.

On the other hand, inspection of the averaged $\theta_{\text {D-D }}$ values displayed in Table 2 indicates that the dihedral angle that connects the two central dendrons are very sensible to the solvent polarity, ranging from $-140.6^{\circ}(\mathrm{DCM})$ to $-151.4^{\circ}(\mathrm{DMF})$ and from $-130.3^{\circ}$ (DMF) to -177.71 (THF) for $6 \mathrm{~T}$ and $18 \mathrm{~T}$, respectively. Also, comparison of the results obtained for the two dendrimers reflects that the conformational fluctuations induced by both thermal and solvent effects are practically independent of the solvent polarity. This feature suggests that solvent effects are mainly caused by unspecific short-range dendrimer..s solvent interactions, which are practically independent of the polarity of solvent molecules. These average values also differ from those derived from QM geometry optimizations in the gas-phase / in DCM solution (i.e. $-163.9^{\circ} /-163.4^{\circ}$ and $163.8^{\circ} /-164.4^{\circ}$ for $6 \mathrm{~T}$ and $18 \mathrm{~T}$, respectively). Similarly, averaged $\theta_{\alpha-\alpha}$ and $\theta_{\alpha-\beta}$ values indicate the influence of the solvent in the conformational dynamics of the two dendrimers, which is also corroborated by the relatively large standard deviations (Table 2). 
The impact of the explicit solvent molecules in the conformation of the dendrimers predicted by QM/MM-MD simulations is fully consistent with observations derived from classical MD simulations. Figure 6 displays the histogram occupancy of $\theta_{\alpha-\alpha}$ and $\theta_{\alpha-\beta}$ for the two examined dendrimers. The lower dispersion of dihedral angles for $6 \mathrm{~T}$ compared to that of $18 \mathrm{~T}$ is also consistent with classical MD results, evidencing the effect of the solvent in the solute dynamics increases with the generation number. Interestingly, histograms displayed in Figure 6 provide detailed information about the influence of the solvent polarity in the conformational variability of the dendrimers. Thus, the narrowest dispersions of $\theta_{\alpha-\alpha}$ and $\theta_{\alpha-\beta}$ were found in DMF, the solvent with highest polarity, for both $6 \mathrm{~T}$ and $18 \mathrm{~T}$, while the widest dispersions of such dihedral angles correspond to DCM, the solvent of lowest polarity. According to these results, the conformational flexibility increases with decreasing solvent polarity, this phenomenon being more pronounced for $18 \mathrm{~T}$ than $6 \mathrm{~T}$.

The averaged electronic properties in DCM obtained using single-point TD-DFT calculations at the B3P86/6-31G(d) level on the snapshots extracted from the last 5 ps of the corresponding QM/MM-MD trajectories are included in Table 1. For both 6T and $18 \mathrm{~T}$ dendrimers, the averaged IP values $(5.85 \pm 0.13$ and $6.03 \pm 0.28 \mathrm{eV}$, respectively) are slightly lower than the ones derived from PCM-TD-DFT (5.94 and $6.09 \mathrm{eV}$, respectively). Although the $\mathrm{QM} / \mathrm{MM}-\mathrm{MD}$ values are apparently higher than the experimental estimations yet (5.63 and $5.52 \mathrm{eV}$, respectively), inspection to the standard deviations indicates a significant improvement with respect to static calculations on a single conformation with an implicit solvent model. Accordingly, average values and their standard deviations point out that the incorporation of effects associated to both the conformational dynamics of the dendrimer and the explicit solvent molecules is strictly necessary. Indeed, the remaining discrepancy between QM/MM-MD and experimental 
estimations must be attributed to two technical factors: 1) the limited length of the QM/MM-MD trajectory (5 ps), and 2) the theoretical framework of the TD-DFT method (B3P86/6-31G(d)). These factors are defined by the computational cost of QM/MM-MD calculations that, in spite of the recent advances in computer science, is huge.

Comparison among the electronic properties derived from QM/MM-MD simulations in DCM, THF and DMF (Table 3) reveals important features that were undetected by QM and QM/MM calculations. Noticeably, both the IP and $\varepsilon_{\mathrm{g}}$ of $18 \mathrm{~T}$ decreases by $\sim 0.3$ $\mathrm{eV}$ when the solvent changes from DCM to DMF or THF. This reduction, which was not observed in $\mathrm{QM}$ and $\mathrm{QM} / \mathrm{MM}$ calculations (i.e. differences were $<0.1 \mathrm{eV}$ in all cases) is consistent with experimental observations. Thus, the $\varepsilon_{\mathrm{g}}$ of $18 \mathrm{~T}$ in THF is smaller than in DCM. Furthermore, the QM/MM-MD $\varepsilon_{\mathrm{g}}$ values in DCM and THF differ by only 0.14 and $0.06 \mathrm{eV}$, respectively, from the experimental estimations. ${ }^{24,68}$ Unfortunately, the IP value of $18 \mathrm{~T}$ in THF is not available. On the other hand, the solvent-induced variability in the electronic properties of 6T predicted by QM/MM-MD simulations is much less pronounced than for $18 \mathrm{~T}$. This must be attributed to the fact that the conformational variability at the branches of $6 \mathrm{~T}$ is considerably lower than at the branches of $18 \mathrm{~T}$, as was previously discussed. Thus, the $\varepsilon_{\mathrm{g}}$ values predicted for $6 \mathrm{~T}$ in DCM and THF are 3.08 and $3.07 \mathrm{eV}$, respectively, while the experimental values are 2.76 and $2.57 \mathrm{eV}$, respectively.

\section{CONCLUSIONS}

We have presented a comprehensive theoretical study to examine the influence of the solvent, flexibility and conformational dynamics on the electronic properties of hyperbranched oligothiophenes. Results indicate that solvent-induced polarization 
effects captured by PCM-DFT and PCM-TD-DFT calculations on energy minimized conformations are very small. Accordingly, application of implicit solvent models on static geometries does not provide any significant change with respect to properties computed in the gas-phase. On the other hand, conformational fluctuations at the branches of the dendrimers captured by MD simulations evidence the remarkable role of the conformational flexibility and dynamics in the structure of the dendrimers. The importance of these phenomena, which are independent of the solvent, increases with the generation number. In spite of this, results derived from QM/MM calculations suggests that the omission of all solute...solvent interactions different from the electrostatic ones affects the reliability of the theoretical model. Finally, results obtained using the QM/MM-MD simulations are of highest quality, allowing not only to consider the effects conformational dynamics and short-range solute $\cdots$ solvent interactions but also to differentiate among the influence of different solvents. Thus, QM/MM-MD simulations indicate that the conformational flexibility of the dendrimers increases with decreasing solvent polarity, even though this phenomenon becomes more important with increasing generation number. The QM/MM-MD model used in this work should be considered as a reference for future theoretical studies of hyperbranched all-Th macromolecules and oligomers.

\section{ACKNOWLEDGEMENTS}

This work was supported by MINECO-FEDER (MAT2015-69367-R) and by the Generalitat de Catalunya (XRQTC). Authors are indebted to the "Centre de Supercomputació de Catalunya" (CESCA) for the computational resources provided. Support for the research of C.A. was received through the prize "ICREA Academia" for excellence in research funded by the Generalitat de Catalunya. 


\section{REFERENCES}

1. E. Buhleier, W. Wehner and F. Vögtle, Synthesis, 1978, 155-158.

2. G. R. Newkome, Z. Yao, G. R. Baker and V. K. Gupta, J. Org. Chem., 1985, 50, 2003-2004.

3. D. A. Tomalia, H. Baker, J. Dewald, M. Hall, G. Kallos, S. Martin, J. Roeck, J. Ryder and P. Smith, Polym. J. (Tokyo), 1985, 17, 117-132.

4. R. G. Weiner and S. E. Skrabalak, Angew. Chem. Int. Ed., 2015, 54, 1181-1184.

5. M. Cano, A. Sanchez-Ferrer, J. L. Serrano, N. Gimeno and M. B. Ros, Angew. Chem. Int. Ed., 2014, 53, 13449-13453.

6. K. Kirkorian, A. Ellis and L J. Twyman, Chem. Soc. Rev., 2012, 41, 6138-6159.

7. B. N. S. Thota, H. V. Berlepsch, C. Bottcher and R. Haag, Chem. Commun., $2015, \mathbf{5 1}, 8648-8651$

8. S. Castelar, J. Barbera, M. Marcos, P. Romero, J. L. Serrano, A. Golemme and R. Termine, J. Phys. Chem. C, 2013, 44, 7321-7332.

9. X. H. Hu, H. Yuan, J. Chang, B. He and Z. W. Gu, Angew. Chem. Int. Ed., 2012, 51, 3130-3133.

10. X. Zhang, Z. J. Zhang, X. H. Xu, Y. K. Li, Y. C. Li, Y. T Jian and Z. W. Gu, Angew. Chem. Int. Ed., 2015, 54, 4289-4294.

11. E. Fedeli, S. Hernandez-Ainsa, A. Lancelot, R. Gozalez-Pastor, P. Calvo, T. Sierra and J. L. Serrano, Soft Matter, 2015, 11, 6009-6017.

12. K. J. Zhou, L. H. Nguyen, J. B. Miller, Y. F. Yan, P. Kos, H. Xiong, L. Li, J. Hao, J. T. Minning, H. Zhu and D. J. Siegwart, Proc. Natl. Acad. Sci., 2016,113, 520-525 
13. S. A. McNelles, S. D. Knight, N. Janzen, J. F. Valliant and A. Adronov, Biomacromolecules, 2015, 16, 3033-3041.

14. L. Z. Zhao, J. Y. Zhu, Y. J. Cheng, Z. J. Xiong, Y. Q. Tang, L. L. Guo, X. Y. Shi and J. H. Zhao, ACS Appl. Mater. Interfaces, 2015, 7, 19798-19808.

15. S. Pin and M. Bai, Chem. Commun., 2012, 48, 9498-9500.

16. S. M. Grayson and J. M. J. Fréchet, Chem. Rev., 2001, 101, 3819-3867.

17. J. Lim, M. Kostiainen, J. Maly, V. C. P. da Costa, O. Annunziata, G. M. Pavan and E. E. Simanek, J. Am. Chem. Soc., 2013, 135, 4660-4663.

18. K. Karatasos, P. Posocco, E. Laurini and S. Pricl, Macromol. Biosci., 2012, 12, 225-240.

19. S. Kanchi, G. Suresh, U. D. Pritakumar, K. G. Ayappa and P. K. Maiti, J. Phys. Chem. B, 2015, 119, 12990-13001.

20. A. Mishra, C. Q. Ma and P. Baeuerle, Chem. Rev., 2009, 109, 1141-1276.

21. C. Xia, X. Fan, J. Locklin and R. C. Advincula, Org. Lett., 2002, 4, 2067-2070.

22. C. Xia, X. Fan, J. Locklin, R. C. Advincula, A. Gies and W. Nonidez, J. Am. Chem. Soc., 2004, 126, 8735-8743.

23. C.-Q. Ma, E. Mena-Osteritz, T. Debaerdemaeker, M. M. Wienk, R. A. Janssen and P. Bäuerle, Angew. Chem. Int. Ed., 2007, 46, 1679-1683.

24. C.-Q. Ma, E. Mena-Osteritz, M. Wunderlin, G. Schulz, P. Bäuerle, Chem. Eur. J., 2012, 18, 12880-12901.

25. A. L. Kanibolotsky, I. F. Perepichka and P. J. Skabara, Chem. Soc. Rev., 2010, 39, 2695-2728.

26. C.-Q. Ma, M. Fonrodona, M. C. Shikora, M. M. Wienk, R. A. J. Janssen and P. Bäuerle, Adv. Funct. Mater., 2008, 18, 3323-3331. 
27. W. W. Wong, C.-Q. Ma, W. Pisula, X. Feng, D. J. Jones, K. Müllen, R. A. J. Janssen, P. Bäuerle and A. B. Holmes, J. Am. Chem. Soc., 2010, 22, 457-466.

28. F. Rodríguez-Ropero, D. Zanuy and C. Alemán, Polymer, 2010, 51, 308-315.

29. E. Córdova-Mateo, F. Rodríguez-Ropero, O. Bertran and C. Alemán, Chem. Phys. Chem., 2012, 13, 1354-1362.

30. E. Córdova-Mateo, O. Bertran, A. D. Schlüter, M. Kröger and C. Alemán, Soft Matter, 2015, 11, 1116-1126.

31. P. Sonar, H. Benmansour, T. Geiger and A. D. Schlüter, Polymer, 2007, 48, 4996-5004.

32. F. Rodríguez-Ropero, D. Zanuy and C. Alemán, Polymer, 2010, 51, 308-315.

33. J. L. Palma, E. Atas, L. Hardison, T. B. Marder, J. C. Collings, A. Beeby, J. S. Mellinger, J. L. Krause, V. D. Kleiman and A. E. Roitberg, J. Phys. Chem. C, 2010, 114, 20702-20712.

34. M. J. Frisch, G. W. Trucks, H. B. Schlegel, G. E. Scuseria, M. A. Robb, J. R. Cheeseman, G. Scalmani, V. Barone, B. Mennucci, G. A. Petersson, H. Nakatsuji, M. Caricato, X. Li, H. P. Hratchian, A. F. Izmaylov, J. Bloino, G. Zheng, J. L. Sonnenberg, M. Hada, M. Ehara, K. Toyota, R. Fukuda, J. Hasegawa, M. Ishida, T. Nakajima, Y. Honda, O. Kitao, H. Nakai, T. Vreven, J. A. Jr Montgomery, J. E. Peralta, F. Ogliaro, M. Bearpark, J. J. Heyd, E. Brothers, K. N. Kudin, V. N. Staroverov, R. Kobayashi, J. Normand, K. Raghavachari, A. Rendell, J. C. Burant, S. S. Iyengar, J. Tomasi, M. Cossi, N. Rega, J. M. Millam, M. Klene, J. E. Knox, J. B. Cross, V. Bakken, C. Adamo, J. Jaramillo, R. Gomperts, R. E. Stratmann, O. Yazyev, A. J. Austin, R. Cammi, C. Pomelli, J. W. Ochterski, R. L. Martin, K. Morokuma, V. G. Zakrzewski, G. A. Voth, P. Salvador, J. J. Dannenberg, S. Dapprich, A. D. Daniels, O. Farkas, J. B. 
Foresman, J. V. Ortiz, J. Cioslowski and D. J. Fox, Gaussian 09, revision A.01, Gaussian, Inc.: Wallingford, CT, 2009.

35. S. Miertus, E. Scrocco and J. Tomasi, J. Chem. Phys., 1981, 55, 117-129.

36. S. Miertus and J. Tomasi, J. Chem. Phys., 1982, 65, 239-245.

37. J. Tomasi, B. Mennucci and R. Cammi, Chem. Rev., 2005, 105, 2999-3094.

38. E. Böes, P. R. Livotto, H. Stassen, Chem. Phys., 2006, 331,142-158.

39. A. D. Becke, J. Chem. Phys., 1993, 98, 1372-1378.

40. C. Lee, W. Yang and R. G. Parr, Phys. Rev. B, 1988, 37, 785-789.

41. P. C. Harihara and J. A. Pople, Theor. Chim. Acta, 1973, 28, 213-222.

42. A. D. McLean and G. S. Chandler, J. Chem. Phys., 1980, 72, 5639-5649.

43. M. J. Frisch, J. A. Pople and J. S. Binkley, J. Chem. Phys., 1984, 80, 3265-3270.

44. G. D. Hawkins, C. J. Cramer and D. G. Truhlar, J. Chem. Phys. B, 1998, 102, 3257.

45. Y. H. Jang, W. A. Goddard III, K. T. Noyes, L. C. Sowers, S. Hwang and D.S. Chung, J. Phys. Chem. B, 2003, 107, 344.

46. J. I. Iribarren, J. Casanovas, D. Zanuy and C. Alemán, Chem. Phys., 2004, 302, 77.

47. J. F. Janak, Phys. Rev. B, 1978, 18, 7165-7168.

48. J. P. Perdew, Density Functional Methods in Physics, ed. R. M. Dreizler and J. Providenca, Plenum Press, New York and London, 1985.

49. M. Levy and A. Nagy, Phys. Rev. A, 1999, 59, 1687-1689.

50. D. Jacquemin, J. Preat, E. Perpète and C. Adamo, Int. J. Quantum Chem., 2010, 110, 2121-2129.

51. J. Preat, D. Jacquemin and E. Perpète, Int. J. Quantum Chem., 2010, 110, 2147 2154. 
52. J. Preat, B. Teixeira-Dias, C. Michaux, E. A. Perpète and C. Alemán, J. Phys. Chem. A, 2011, 115, 13642-13648.

53. J. P. Perdew, Phys. Rev. B, 1986, 33, 8822-8824.

54. J. P. Perdew, K. Burke and Y. Wang, Phys. Rev. B, 1996, 54, 16533-16539.

55. L. Kale, R. Skeel, M. Bhandarkar, R. Brunner, A. Gursoy, N. Krawtz, J. Phillips, A. Shinozaki, K. Varadarajan and K. J. Schulten, Comput. Phys., 1999, 151, 283. 56. W. D. Cornell, P. Cieplak, C. I. Bayly, I. R. Gould, K. M. Merz, D. M. Ferguson, D. C. Spellmeyer, T. Fox, J. W. Caldwell and P. A. Kollman, J. Am. Chem. Soc., 1995, 117, 5179-5197.

57. J. R. Blas, M. Marquez, J. L. Sessler, F. J. Luque and M. Orozco, J. Am. Chem. Soc., 2002, 124, 12796.

58. F. Rodriguez-Ropero, J. Preat, D. Zanuy, J. Torras and C. Alemán, J. Phys. Chem. B, 2009, 113, 8284-8287.

59. C. J. Shih, S. Lin, M. S. Strano and D. Blankschtein, J. Am. Chem. Soc., 2010, 132, $14638-14648$

60. J. Wang, R. M. Wolf, J.W. Caldwell and D. A. Case, J. Comput. Chem., 2004, 15, $1157-1174$.

61. P. Cieplak, W. Cornell, C. I. Bayly and P. A. Kollman, J. Comput. Chem., 1995, 16, 1357.

62. J. Torras, G. d. M. Seabra, E. Deumens, S. B. Trickey and A. E. A. Roitberg, J. Comput. Chem., 2008, 29, 1564-1573.

63. D. A. Case, V. Babin, J. T. Berryman, R. M. Betz, Q. Cai, D. S. Cerutti, T. E. Cheatham III, T. A. Darden, R. E. Duke, H. Gohlke, A. W. Goetz, S. Gusarov, N. Homeyer, P. Janowski, J. Kaus, I. Kolossváry, A. Kovalenko, T. S. Lee, S . LeGrand, T. Luchko, R. Luo, B. Madej, K. M. Merz, F. Paesani, D. R. Roe, A. 
Roitberg, C. Sagui, R. Salomon-Ferrer, G. Seabra, C. L. Simmerling, W. Smith, J. Swails, R. C. Walker, J. Wang, R. M. Wolf, X. Wu and P. A. Kollman, AMBER 14, University of California, San Francisco, 2014.

64. B. Z. Zhang, R. Wepf, M. Kröger, A. Halperin and A. D. Schlüter, Macromolecules, 2011, 44, 6785.

65. B. Z. Zhang, R. Wepf, K. Fischer, M. Schmidt, S. Besse, P. Lindner, B. King, R. Sigel, P. Schurtenberger, Y. Talmon, Y. Ding, M. Kröger, A. Halperin and A. D. Schlüter, Angew. Chem., Int. Ed., 2011, 50, 737.

66. O. Bertran, B. Z. Zhang, A. D. Schlüter, A. Halperin, M. Kröger and C. Alemán, RSC Adv., 2013, 3, 126.

67. O. Bertran, B. Zhang, A. D. Schlüter, M. Kröger and C. Alemán, J. Phys. Chem. $B, 2013,117,6007$.

68. E. Badaeva, M. R. Haspham, R. Guda, O. Süzer, C.-Q. Ma, P. Bäuerle, T. Goodson III and S. Tretiak, J. Phys. Chem. B, 2010, 114, 15808-15817. 
Table 1. Comparison between the IP and $\varepsilon_{\mathrm{g}}$ values determined by $\mathrm{CV}$ for $6 \mathrm{~T}$ and $18 \mathrm{~T}$ in DCM solution and the estimations derived from the different theoretical approximations used in this work.

\begin{tabular}{lcccc}
\hline & \multicolumn{2}{c}{ 6 T } & \multicolumn{2}{c}{ 18T } \\
& IP $(\mathbf{e V})$ & $\boldsymbol{\varepsilon}_{\mathbf{g}}(\mathbf{e V})$ & $\mathbf{I P}(\mathbf{e V})$ & $\boldsymbol{\varepsilon}_{\mathbf{g}}(\mathbf{e V})$ \\
\hline PCM-DFT [B3LYP/6-31G(d)] & 5.24 & 3.30 & 4.98 & 2.65 \\
PCM-TD-DFT [B3P86/6-31G(d)] & 5.94 & 2.94 & 5.69 & 2.28 \\
$\begin{array}{l}\text { QM/MM [B3LYP/6-31G(d) for } n \text { T } \\
\text { and point charges for solvent }\end{array}$ & 5.94 & 3.01 & 6.06 & 2.33 \\
molecules] & & & & \\
$\begin{array}{l}\text { QM/MM-MD [TD-DFT B3P86/6- } \\
\text { 31G(d)] explicit solvent molecules }\end{array}$ & $5.85 \pm 0.13$ & $3.08 \pm 0.17$ & $6.03 \pm 0.28$ & $2.49 \pm 0.23$ \\
$\begin{array}{l}\text { UV-vis spectroscopy and cyclic } \\
\text { voltammetry in DCM }\end{array}$ & 5.63 & 2.76 & 5.52 & 2.35 \\
\hline
\end{tabular}

${ }^{\mathrm{a}}$ From reference 24. 
Table 2. Averaged geometries derived from hybrid QM/MM-MD simulation in solution (i.e. DCM, DMF, and THF) using explicit solvent in the framework of the B3LYP/3-21G(d) method. Standard deviations are also shown (parenthesis).

\begin{tabular}{|c|c|c|c|c|c|c|c|}
\hline \multicolumn{2}{|l|}{ System } & \multirow{2}{*}{$\begin{array}{c}\theta_{\alpha-\alpha}\left({ }^{\circ}\right) \\
135.5(15.8)\end{array}$} & \multirow{2}{*}{$\begin{array}{c}\boldsymbol{\theta}_{\boldsymbol{\alpha}-\boldsymbol{\beta}}\left(^{\mathbf{0}}\right) \\
-33.7(27.1)\end{array}$} & \multirow{2}{*}{$\begin{array}{c}\mathbf{R}_{\boldsymbol{\alpha - \alpha}}(\AA) \\
1.458(0.028)\end{array}$} & \multirow{2}{*}{$\begin{array}{c}\mathbf{R}_{\alpha-\beta}(\AA) \\
1.468(0.028)\end{array}$} & \multirow{2}{*}{$\begin{array}{c}\mathbf{R}_{\mathbf{D}-\mathbf{D}}(\AA) \\
1.455(0.028)\end{array}$} & \multirow{2}{*}{$\begin{array}{c}\theta_{\text {D-D }}\left({ }^{\mathbf{o}}\right) \\
-140.6(9.9)\end{array}$} \\
\hline & $\mathrm{DCM}$ & & & & & & \\
\hline \multirow[t]{2}{*}{$6 \mathrm{~T}$} & DMF & $209.8(11.0)$ & $-95.2(15.9)$ & $1.454(0.030)$ & $1.474(0.029)$ & $1.449(0.028)$ & $-151.4(21.1)$ \\
\hline & THF & $148.9(16.3)$ & $-60.5(31.2)$ & $1.458(0.030)$ & $1.473(0.030)$ & $1.453(0.028)$ & $-147.7(9.4)$ \\
\hline \multicolumn{2}{|c|}{ QM Gas-phase ${ }^{(a)}$} & 131.1 & -41.7 & 1.456 & 1.466 & 1.447 & -163.9 \\
\hline \multicolumn{2}{|c|}{ QM DCM ${ }^{(b)}$} & 131.1 & -41.8 & 1.457 & 1.466 & 1.447 & -163.4 \\
\hline \multirow{3}{*}{$18 \mathrm{~T}$} & $\mathrm{DCM}$ & $140.0(54.2)$ & $-108.0(91.0)$ & $1.453(0.030)$ & $1.471(0.027)$ & $1.449(0.030)$ & $-161.2(12.2)$ \\
\hline & DMF & $174.5(25.9)$ & $-61.2(24.5)$ & $1.451(0.031)$ & $1.465(0.031)$ & $1.450(0.027)$ & $-139.3(10.5)$ \\
\hline & THF & $179.9(37.6)$ & $-78.3(44.8)$ & $1.451(0.030)$ & $1.469(0.030)$ & $1.448(0.028)$ & $-177.7(9.0)$ \\
\hline \multicolumn{2}{|c|}{ QM Gas-phase ${ }^{(a)}$} & 132.2 & -41.3 & 1.455 & 1.465 & 1.445 & -163.8 \\
\hline \multicolumn{2}{|c|}{ QM DCM ${ }^{(\mathrm{b})}$} & 134.8 & -41.5 & 1.452 & 1.464 & 1.447 & -164.4 \\
\hline
\end{tabular}

${ }^{\mathrm{a}}$ From reference 29. ${ }^{(b)}$ Geometry optimization in DCM using the PMC-B3LYP/6-31G(d) method. 
Table 3. Comparison between the IP and $\varepsilon_{\mathrm{g}}$ values determined by QM/MM-MD for 6T and $18 \mathrm{~T}$ in DCM, DMF and THF solutions. Experimental values (when available) are displayed in parenthesis.

\begin{tabular}{ccccc}
\hline & \multicolumn{2}{c}{$\mathbf{6 T}$} & \multicolumn{2}{c}{$\mathbf{1 8 T}$} \\
& $\mathbf{I P}(\mathbf{e V})$ & $\boldsymbol{\varepsilon}_{\mathbf{g}}(\mathbf{e V})$ & $\mathbf{I P}(\mathbf{e V})$ & $\boldsymbol{\varepsilon}_{\mathbf{g}}(\mathbf{e V})$ \\
\hline Dichloromethane (DCM) & $5.85 \pm 0.13$ & $3.08 \pm 0.17$ & $6.03 \pm 0.28$ & $2.49 \pm 0.23$ \\
& $(5.63)^{\mathrm{a}}$ & $(2.76)^{\mathrm{a}}$ & $(5.52)^{\mathrm{a}}$ & $(2.35)^{\mathrm{a}}$ \\
Dimethylformamide (DMF) & $5.91 \pm 0.19$ & $3.02 \pm 0.14$ & $5.47 \pm 0.19$ & $2.17 \pm 0.16$ \\
& & & $(5.52)^{\mathrm{b}}$ & $(2.35)^{\mathrm{b}}$ \\
Tetrahydrofurane (THF) & $5.96 \pm 0.28$ & $3.07 \pm 0.15$ & $5.48 \pm 0.18$ & $2.20 \pm 0.13$ \\
& & $(2.57)^{\mathrm{c}}$ & & $(2.26)^{\mathrm{c}}$ \\
\hline
\end{tabular}

\footnotetext{
${ }^{a}$ Experimental values in DCM taken from reference $24 .{ }^{b}$ Experimental values in DMF, which are identical to those obtained in DCM, taken from reference $24{ }^{\mathrm{c}}$ Experimental values in THF taken from reference 69.
} 


\section{CAPTIONS TO FIGURES}

Figure 1. (a) Schematic picture of dendrimers (left) and dendrons (right). $g$ represents dendrimer generation (from 0 to 2). (b) Definition of structural parameters used in this work: bond distances $\left(R_{D-D}, R_{\alpha-\alpha}\right.$ and $\left.R_{\alpha-\beta}\right)$ and dihedral angles $\left(\theta_{D-D}, \theta_{\alpha-\alpha}\right.$ and $\left.\theta_{\alpha-\beta}\right)$ associated to the rotation of such bonds.

Figure 2. (a) Variation of the (a) IP and (b) $\varepsilon_{\mathrm{g}}$ against $1 / n$, where $n$ is the number of Th rings. Values derived from PCM-DFT and PCM-TD-DFT calculations in DCM and from experiments (UV-vis spectroscopy and CV) in DCM $(n=3,6,9,21,42$ and 45) or $\operatorname{DMF}(n=18$ and 90). Experimental values were taken from reference 24.

Figure 3. HOMO and LUMO frontier molecular orbitals $6 \mathrm{~T}$ calculated in the gasphase (B3LYP/6-31G(d)) and in DCM, THF and DMF solutions (PCM-B3LYP/6$31 G(d))$.

Figure 4. Distribution of solvent molecules as a function of the distance from the geometric center of the Th rings for 6T. The $g(r)$ were calculated considering: (a) the center of masses, the chlorine atoms and the carbon atoms of the DCM molecules; (b) the center of masses, the oxygen atom and the carbon atoms of the THF molecules; and (c) the center of masses, the nitrogen atom and the oxygen atom of the DMF molecules.

Figure 5. Superimposed structures of $18 \mathrm{~T}$ extracted from MD trajectories in DCM (red), THF (blue) and DMF (green) after (a) $5 \mathrm{ns,} \mathrm{(b)} 50 \mathrm{~ns}$ and (c) $100 \mathrm{ns.}$

Figure 6. Dihedral angle histograms $\left(\theta_{\alpha-\alpha}\right.$ and $\left.\theta_{\alpha-\beta}\right)$ for (a) 6T and (b) $18 \mathrm{~T}$ in DCM DMF and THF. Data extracted from QM/MM-MD trajectories. 
(a)
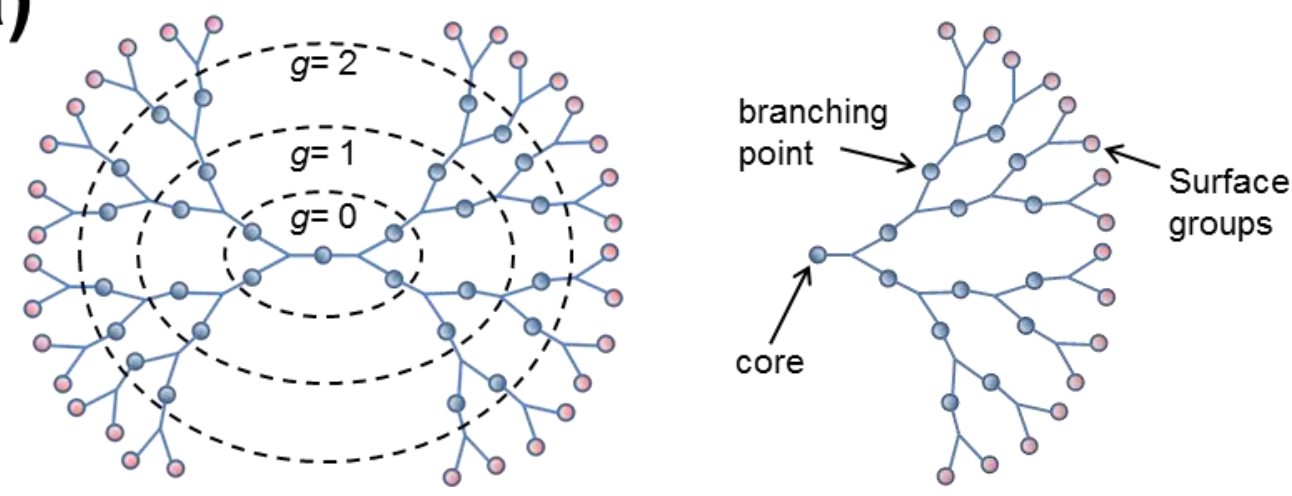

(b)

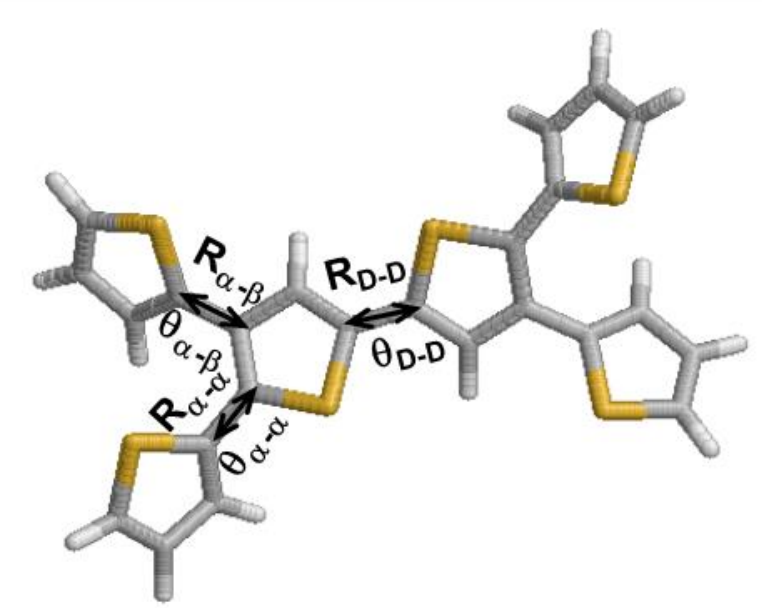

Figure 1 
(a)

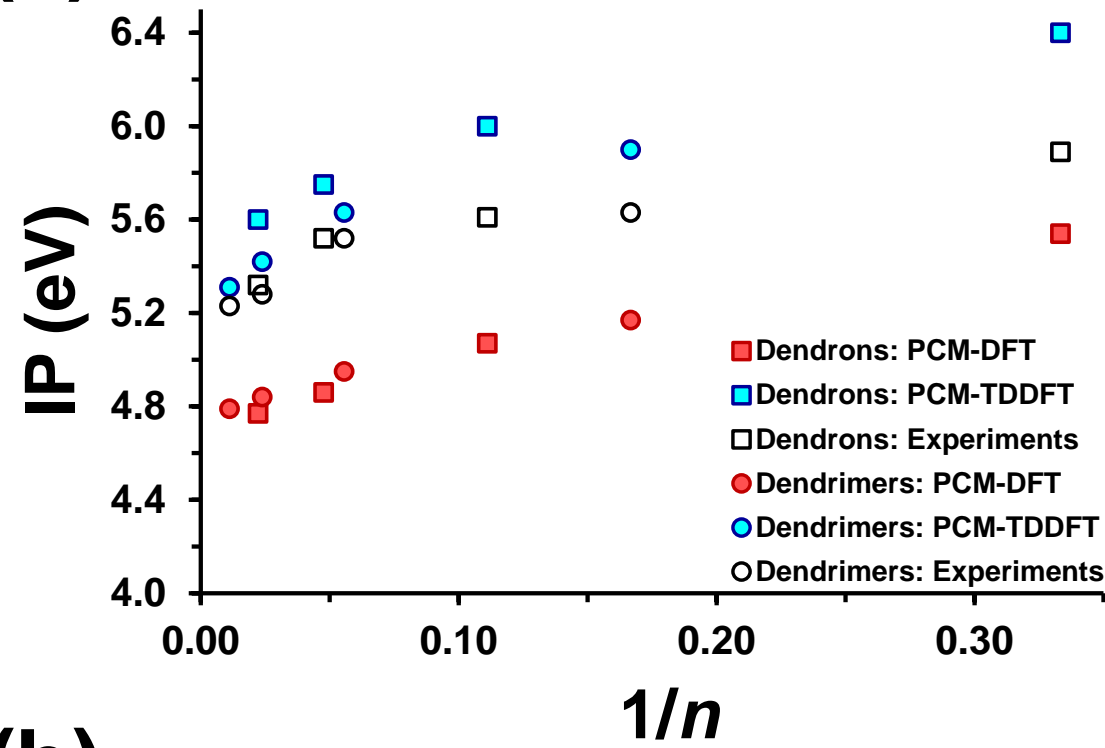

(b)

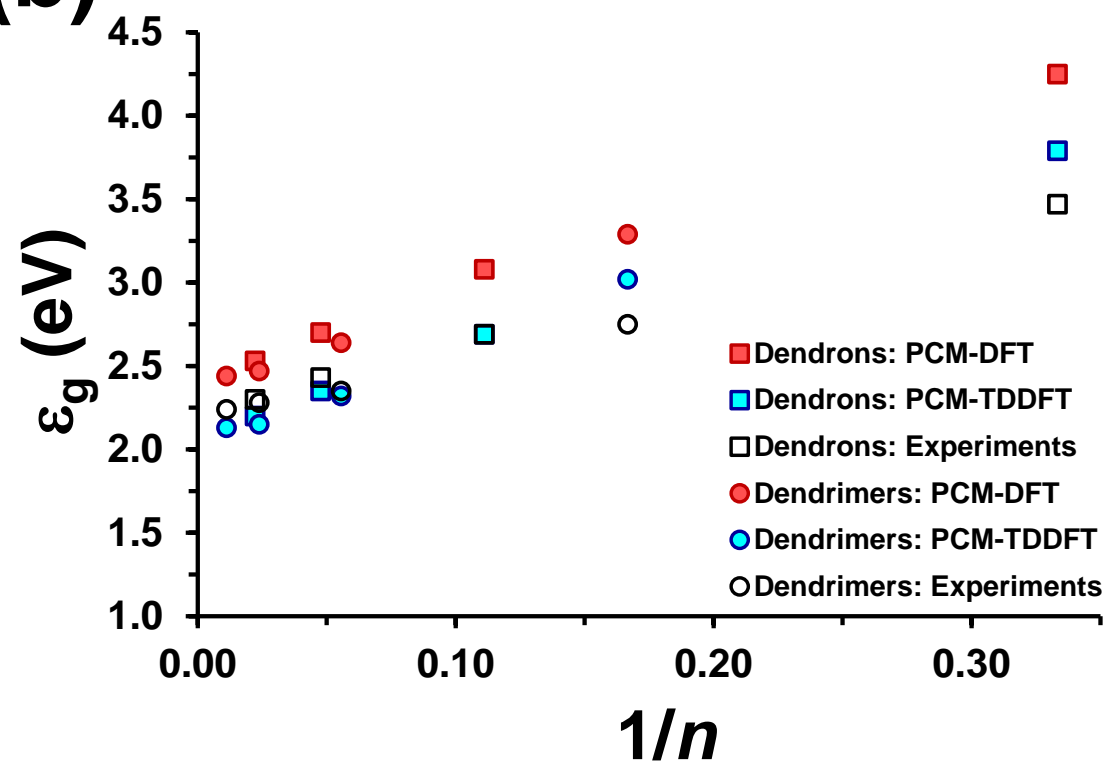

Figure 2 


\section{HOMO}

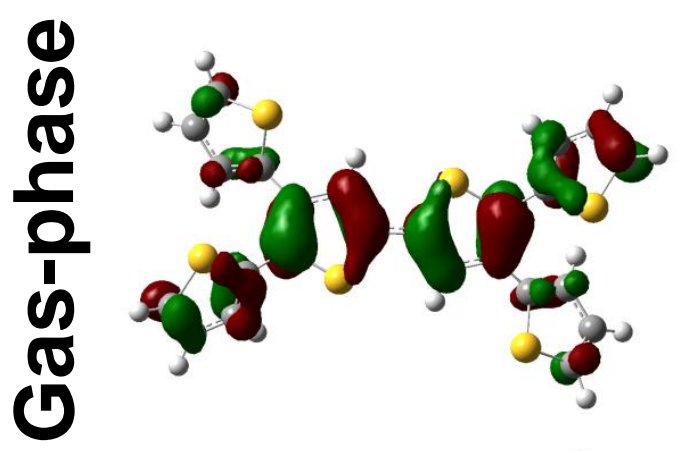

约

늘

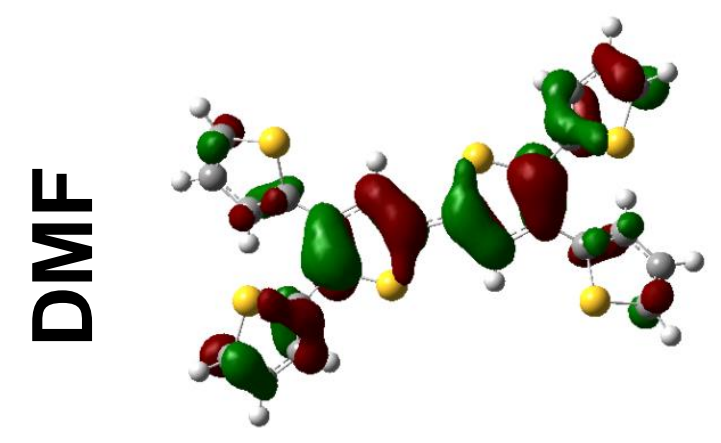

\section{LUMO}
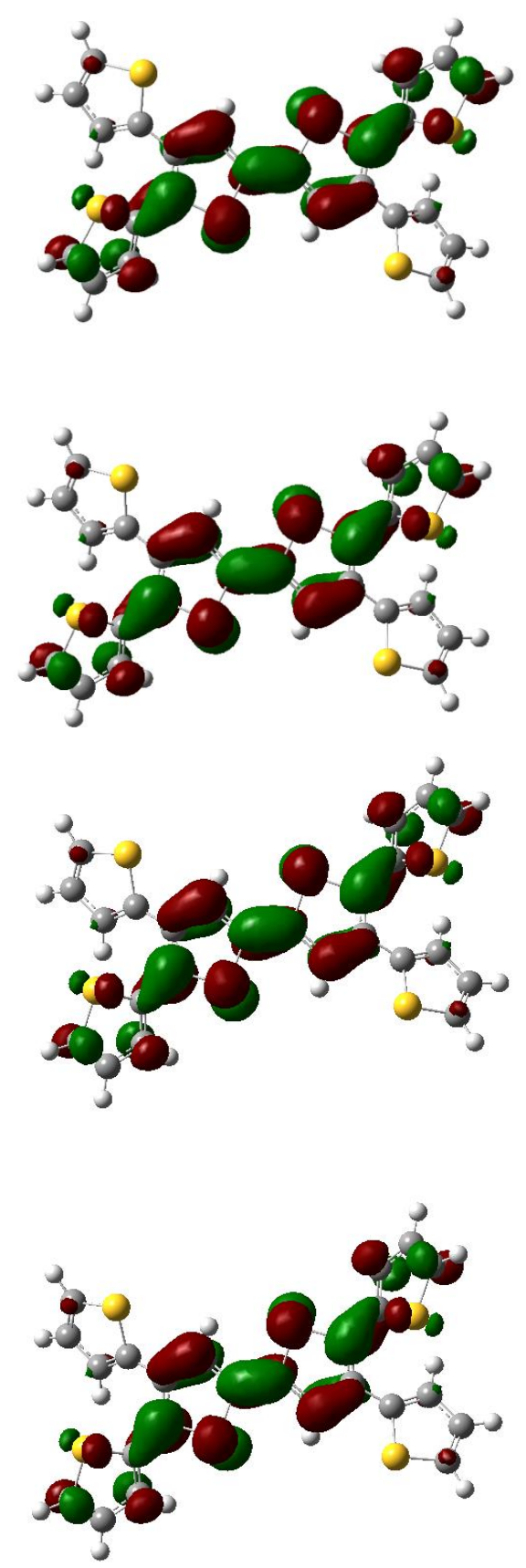

Figure 3 
(a)

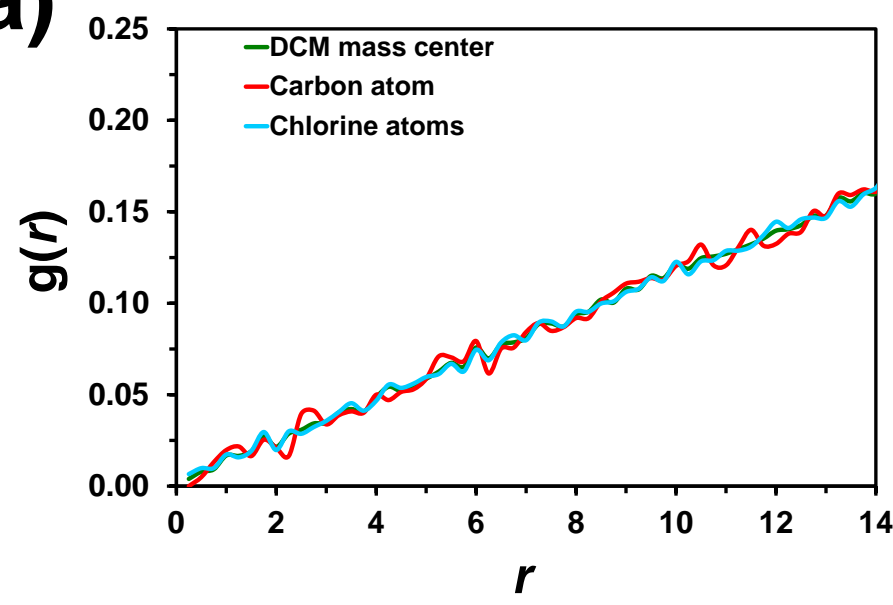

(b)

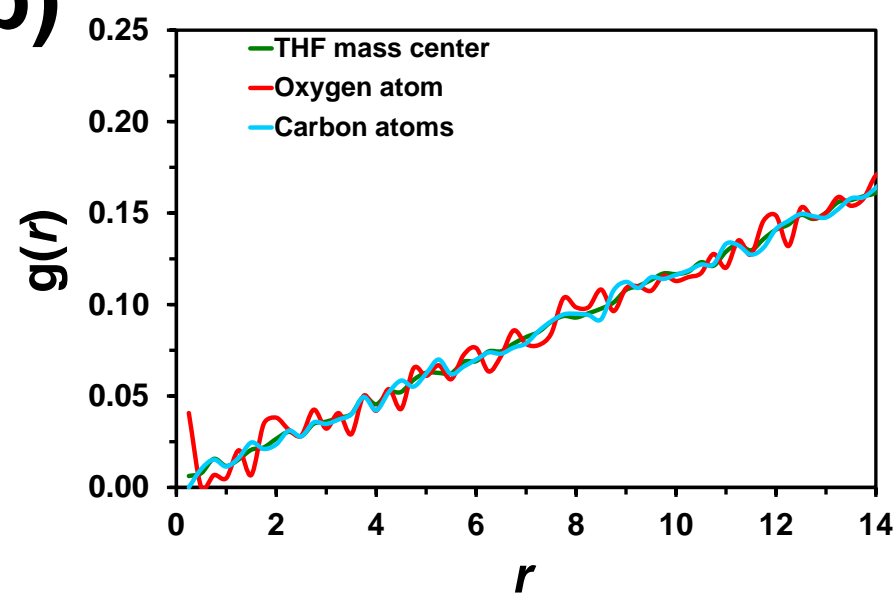

(c)

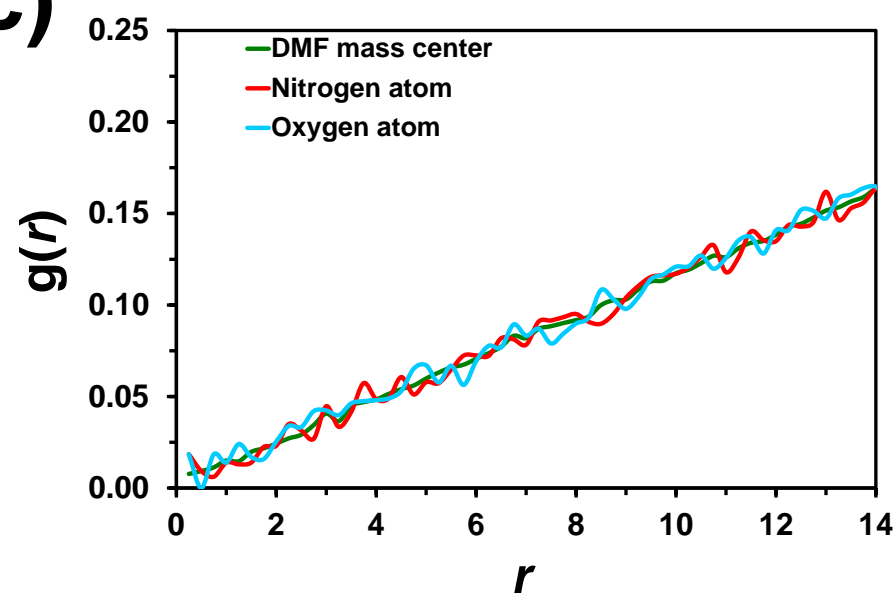

Figure 4 
(a)

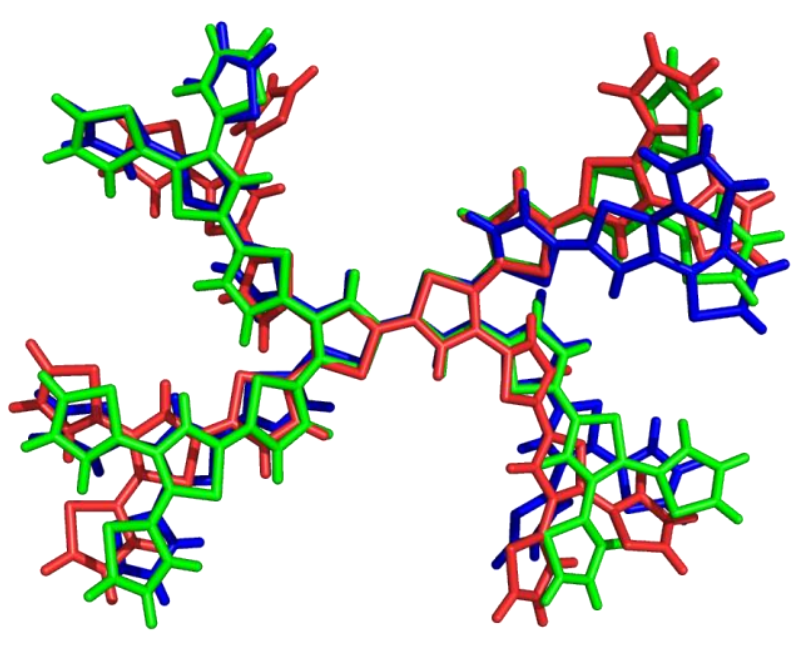

(b)

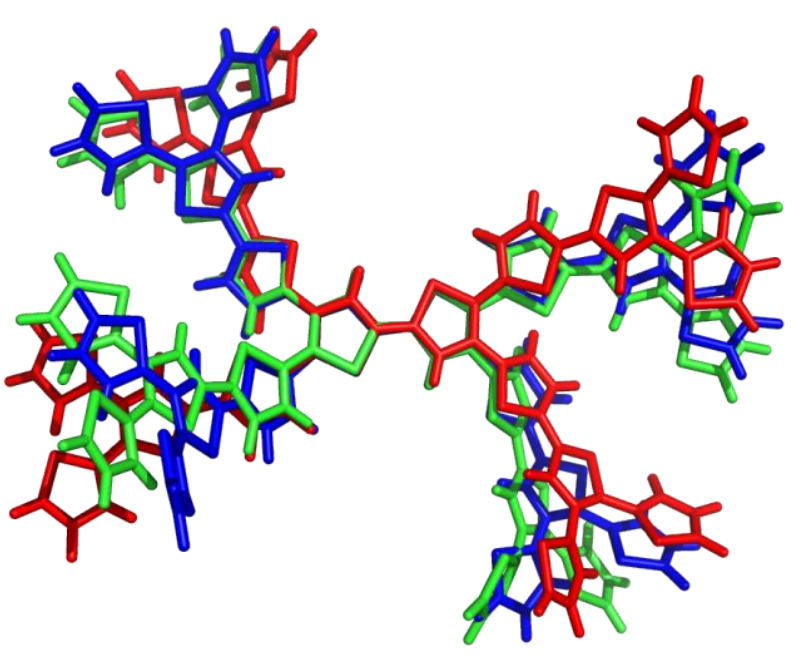

(c)

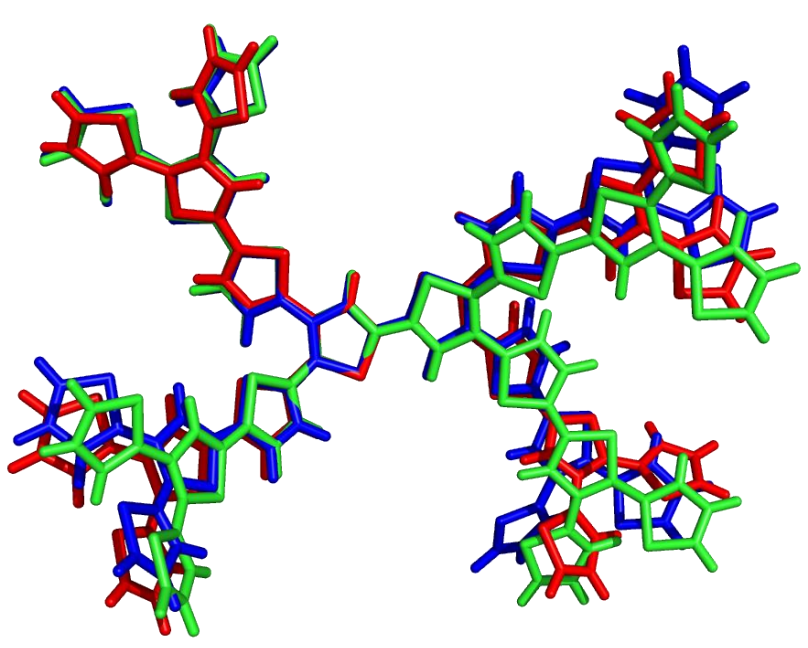

Figure 5 
(a)
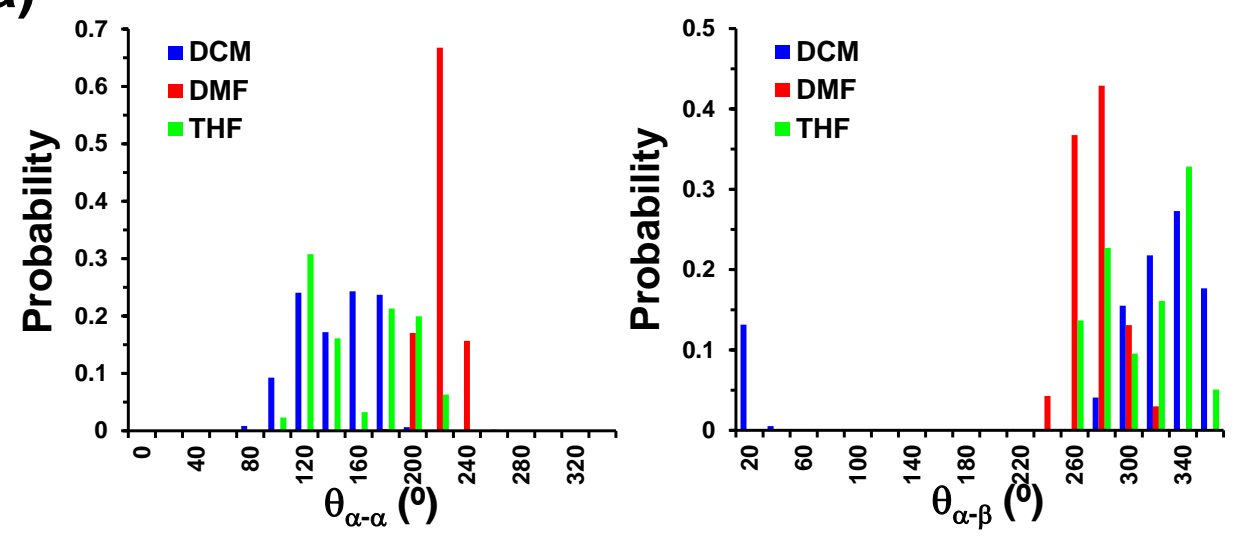

(a)
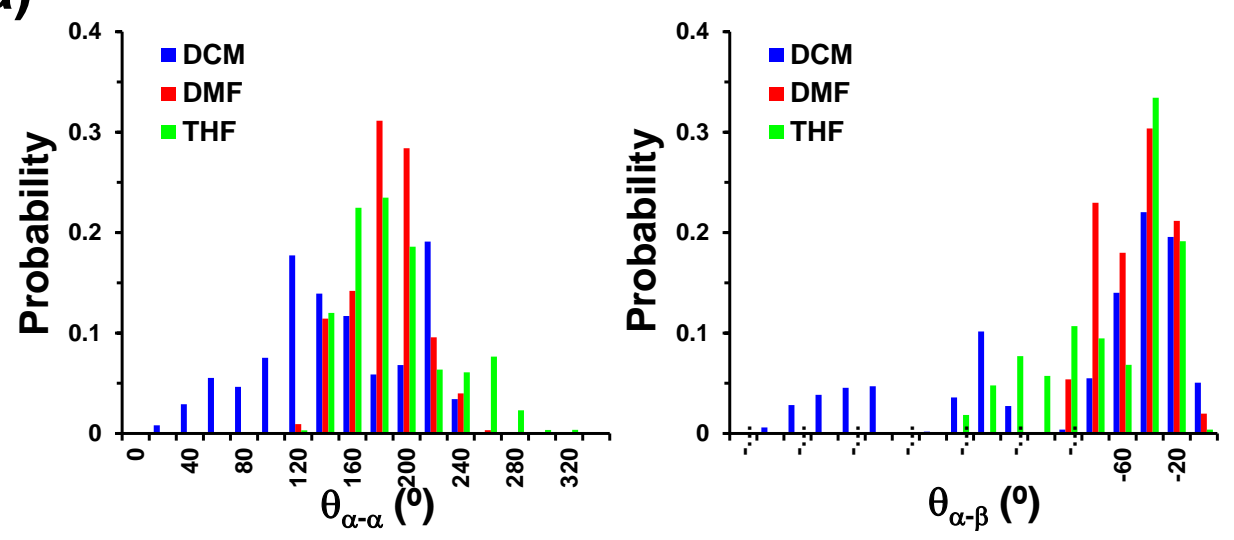

Figure 6 


\section{Graphical Abstract}

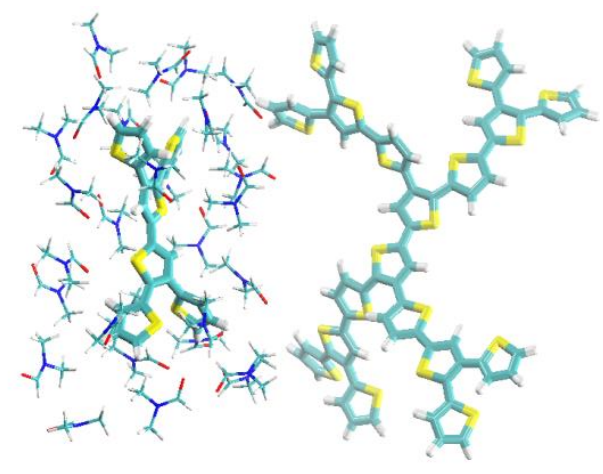

Text: QM/MM-MD simulations of dendrimers with explicit solvent molecules capture the conformational flexibility and microfluctuations induced by different types of solvents. 